\title{
Tumor suppressor Prdx1 is a prognostic factor in esophageal squamous cell carcinoma patients
}

\author{
ISAMU HOSHINO, HISAHIRO MATSUBARA, YASUNORI AKUTSU, \\ TAKANORI NISHIMORI, YASUO YONEYAMA, KENTARO MURAKAMI, \\ HARUHITO SAKATA, KAZUYUKI MATSUSHITA and TAKENORI OCHIAI \\ Department of Frontier Surgery, Graduate School of Medicine, Chiba University, Chiba, Japan
}

Received April 25, 2007; Accepted July 2, 2007

\begin{abstract}
Peroxiredoxins (Prdxs) are a family of antioxidant enzymes that are also known as scavengers of peroxide in mammalian cells. Some reports have shown that the overexpression of Prdx1, which is one of the peroxiredoxins that is a ubiquitously expressed protein, was related to a poor prognosis in several types of human cancers. In this study, we investigated the expression levels of Prdx1 in esophageal squamous cell carcinoma by immunohistochemistry, and the correlation between the Prdx1 expression and the clinical status was elucidated. Immunohistochemical staining was performed in 114 samples which were collected from surgical esophageal cancer specimens. Cytoplasmic staining of Prdx1 was evaluated based on the following scoring criteria: Grade I, negative or weak staining; Grade II, moderate staining; and Grade III, strong staining. The percentage of patients with a Grade I expression of Prx 1 was $20 \%$ (23 of 114), $44 \%$ had Grade II (50 of 114), and 36\% had Grade III (41 of 114). The Prdx1 immunoreactivity showed an inverse significant correlation with $\mathrm{T}$-category $(\mathrm{P}<0.0001)$, lymph node metastasis $(\mathrm{P}=0.048)$, and stage $(\mathrm{P}=0.001)$. In addition, the patients with tumors exhibiting a reduced Prdx 1 expression had shorter overall survival $(\mathrm{P}=0.022)$ in comparison to the patients with tumors which had a higher Prdx1 expression. Currently, Prdx1 has been shown to act as a tumor suppressor. Our results provide strong evidence that the reduced $\operatorname{Prdx} 1$ expression is an important factor in esophageal squamous cancer progression and could serve as a useful prognostic marker.
\end{abstract}

\section{Introduction}

Esophageal cancer is the eighth most frequent cancer and the sixth most frequent cause of death from malignant disease in

Correspondence to: Dr Hisahiro Matsubara, Department of Frontier Surgery, Graduate School of Medicine, Chiba University, Inohana 1-8-1, Chuo-ku, Chiba 260-8670, Japan

E-mail:matsuhm@faculty.chiba-u.jp

Key words: Prdx1, tumor suppressor, esophageal squamous cell carcinoma the world (1). Esophageal squamous cell carcinoma (ESCC) is the most common type in Japan (2). A large number of reports about genetic changes in ESCC have already been published, but little is known about the major tumor suppressor genes or major oncogenes in the process of tumor progression of this malignant disease. ESCC is still a fatal malignancy with a 5 -year rate of $5 \%$ to $20 \%$ for advanced stage patients undergoing a curative resection (3). This miserable prognosis for ESCC involves not only the aggressive character of this tumor but also the limited number of useful markers available for diagnostic purposes.

Therefore, better markers which indicate the malignant potential of ESCC should help in the prognosis or optimal treatment of the patients suffering from this disease. In this study, we focus on Prdx1, one of the peroxiredoxins (Prdxs) belonging to a novel antioxidant family, which has been reported to be a tumor suppressor gene. Prdx 1 has been thought to have an inhibitory function for both c-Abl and c-Myc, of which active forms cause several neoplasms $(4,5)$. Neumann et al (6) showed, using $\operatorname{Prdx} 1$ knockout mice, that $\operatorname{Prdx} 1$ expression correlated with the reactive oxygen species and the incidence of malignant disease. Moreover, our previous study using a cDNA microarray showed that one of the novel histone deacetylase (HDAC) inhibitors, FK228, induced the $\operatorname{Prdx} 1$ expression in the esophageal cancer cell lines and that the expression was associated with the cell toxicity of FK228 (7). The deregulation of HDACs can cause malignant diseases. The inhibition of HDACs is an emerging new strategy in human cancer therapy (8-10). HDAC inhibitors have been shown to induce cell cycle arrest, differentiation, and apoptosis in malignant cells $(9,10)$. From these reports and our former study, Prdx 1 appears to have a possible correlation with an anti-tumor effect by producing epigenetic changes in cancer patients. On the other hand, some researchers have mentioned that this protein may be involved with cell proliferation and tumor growth in several kinds of solid cancers (11-15).

In order to contribute to the controversial discussion about the influence of Prdx 1 expression in malignant tumors, we examined the expression of this protein in 114 cases of ESCC by immunohistochemistry and evaluated the correlations with the clinical parameters of ESCC to determine whether or not the expression of PrdxI in ESCC plays an important role in tumor progression. 
Table I. Characteristics of esophageal squamous cell carcinoma patients.

\section{Variables}

Age (years)

Mean

Range

$\operatorname{Sex}(\mathrm{F} / \mathrm{M})$

Depth of invasion

$\mathrm{T} 1$

$\mathrm{T} 2$

T3

T4

16

50

Lymph node metastasis

N0

55

N1

Distant metastasis

M0

M1

Stage

$$
\text { I }
$$

IIa

IIb

III

IV

Histopathological grading

$$
1
$$$$
2
$$

\section{Materials and methods}

Patients and tissue samples. A total of 114 primary esophageal squamous cancer samples were obtained at the Department of Frontier surgery, Chiba University Hospital, Chiba, Japan. All of the patients were undergoing surgery without any preoperative radiotherapy or chemotherapy. The histological diagnoses revealed that all of the patients had squamous cell carcinoma. Simultaneously normal samples from areas adjacent to the cancerous areas were obtained and they were confirmed to be normal specimens. The clinicopathological characteristics of the samples are listed in Table I. The staging of the tumors was carried out according to the TNM classification.

Immunohistochemistry. The specimens were immersed in $10 \%$ formaldehyde immediately after removal and then were embedded in a paraffin block. Blocks containing both carcinoma and the adjacent normal epithelium were chosen and two serial sections were made from the blocks. One section was stained with hematoxylin and eosin while the
Table II. Immunohistochemistry of Prdx 1 expression in esophageal squamous cell cancer tissue and association with

\begin{tabular}{|c|c|c|c|c|}
\hline \multirow[t]{2}{*}{ Parameters } & \multicolumn{3}{|c|}{ Prdx1 expression level } & \multirow[t]{2}{*}{ P-value } \\
\hline & I & II & III & \\
\hline \multicolumn{5}{|l|}{ Age } \\
\hline$>60$ years & 14 & 30 & 23 & 0.908 \\
\hline$<60$ years & 9 & 20 & 18 & \\
\hline \multicolumn{5}{|l|}{ Sex } \\
\hline Male & 16 & 43 & 36 & 0.136 \\
\hline Female & 7 & 7 & 5 & \\
\hline \multicolumn{5}{|c|}{ Depth of invasion } \\
\hline $\mathrm{T} 1+\mathrm{T} 2$ & 8 & 19 & 37 & $<0.0001$ \\
\hline $\mathrm{T} 3+\mathrm{T} 4$ & 15 & 31 & 4 & \\
\hline \multicolumn{5}{|c|}{ Lymph node metastasis } \\
\hline N0 & 10 & 19 & 26 & 0.048 \\
\hline N1 & 13 & 31 & 15 & \\
\hline \multicolumn{5}{|c|}{ Distant metastasis } \\
\hline M0 & 19 & 38 & 35 & 0.686 \\
\hline M1 & 4 & 12 & 6 & \\
\hline \multicolumn{5}{|l|}{ Stage } \\
\hline $\mathrm{I}+\mathrm{II}$ & 11 & 24 & 35 & 0.001 \\
\hline III + IV & 12 & 26 & 6 & \\
\hline \multicolumn{5}{|c|}{ Histopathological grading } \\
\hline 1 & 12 & 11 & 7 & 0.054 \\
\hline 2 & 9 & 30 & 27 & \\
\hline 3 & 1 & 9 & 8 & \\
\hline
\end{tabular}
clinicopathological parameters.

other section was used for immunohistochemical studies of Prdx1. Immunohistochemical staining was performed in order to detect the expression of Prdx 1 with rabbit antihuman Prdx1 (Alexis Biochemicals, Lausen, Switzerland). In brief, the sections were deparaffinized and the endogenous peroxide activity was inactivated in $100 \%$ methanol containing $3 \%$ hydrogen peroxide. After the blocking of non-specific binding by treating the slides with $5 \%$ skim milk at $37^{\circ} \mathrm{C}$ for $60 \mathrm{~min}$, the slides were incubated at room temperature with a primary antibody at 1:150 dilution. The sections were then washed and incubated with the ENVISION+ kit (Dako, Copenhagen, Denmark) for $60 \mathrm{~min} .3$, 3-Diaminobenzidine was used as a chromogen to reveal the antigen, and the sections were then counterstained with Harris hematoxylin.

Scoring of antibody staining. The Prdx1 immunostaining was semiquantified by a visual grading system (16) in which the intensity of the staining was classified into 3 groups as follows: Grade I, negative or weak staining (Fig. 1A and B); Grade II, moderate staining (Fig. 1C and D); and Grade III, strong staining (Fig. 1E and F). 


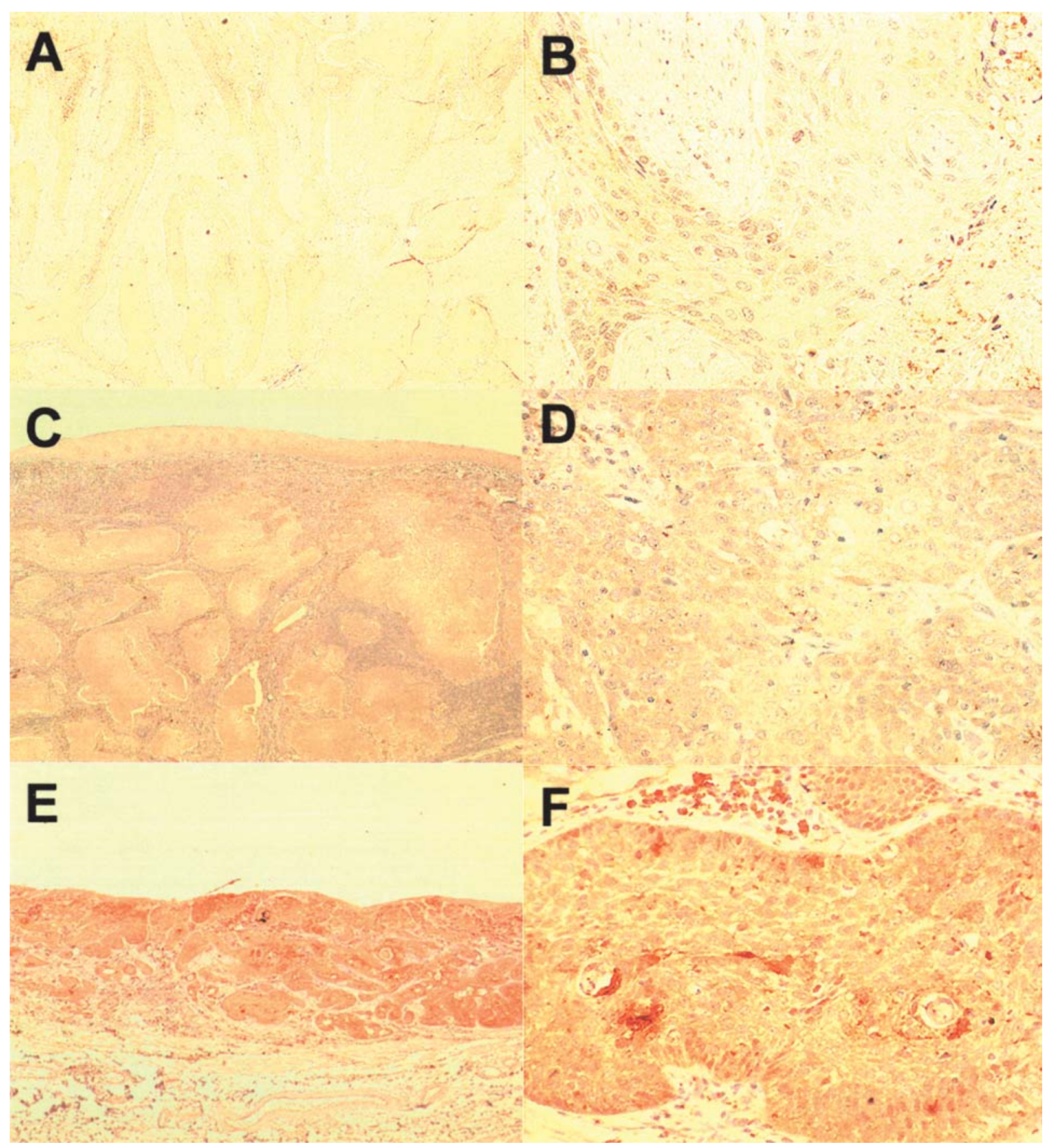

Figure 1. Immunohistochemistry of Prdx 1 in esophageal squamous cell carcinoma specimens. In cancer cells, Prdx1 was demonstrated in the cytoplasm. A, weak staining of Prdx1 (x40). B, magnified view (x200). C, moderate staining of Prdx1 (x40). D, magnified view (x200). E, strong staining of Prdx1 (x40). $\mathrm{F}$, magnified view (x200).

Statistical analysis. The StatView statistical package (SAS Institute Inc., Cary, NC) was used for the statistical analysis. Significant differences between the groups were analyzed using the Chi-square test. The survival data of patients were analyzed using the Kaplan-Meier estimation method and the survival curves were evaluated with the log-rank test. A value of $\mathrm{P}<0.05$ was considered to be statistically significant.

\section{Results}

Immunohistological analysis of Prdx1 expression in ESCC. By immunohistochemistry, the Prdx1 expression in the cytoplasm was observed in $90 \%$ of the specimens examined. The percentage of patients with Grade I expression was $20 \%$ (23 of $114), 44 \%$ had Grade II (50 of 114), and $36 \%$ had Grade III (41 of 114). In order to simplify the correlation of the Prdx1 expression with the clinical features, T-categories were divided into $\mathrm{T} 1+\mathrm{T} 2$ and $\mathrm{T} 3+\mathrm{T} 4$ groups .

Comparing the expression level of Prdx 1 with clinical features, the high expression groups included significantly more $\mathrm{T} 1+\mathrm{T} 2$, N0 and stage I + II case patients. There were significant differences in the expression level of $\operatorname{Prdx} 1$ between the $\mathrm{T} 1+\mathrm{T} 2$ and $\mathrm{T} 3+\mathrm{T} 4$ groups $(\mathrm{P}<0.0001)$, between the stage I + II and stage III + IV $(\mathrm{P}=0.001<0.01)$ groups, and 
A

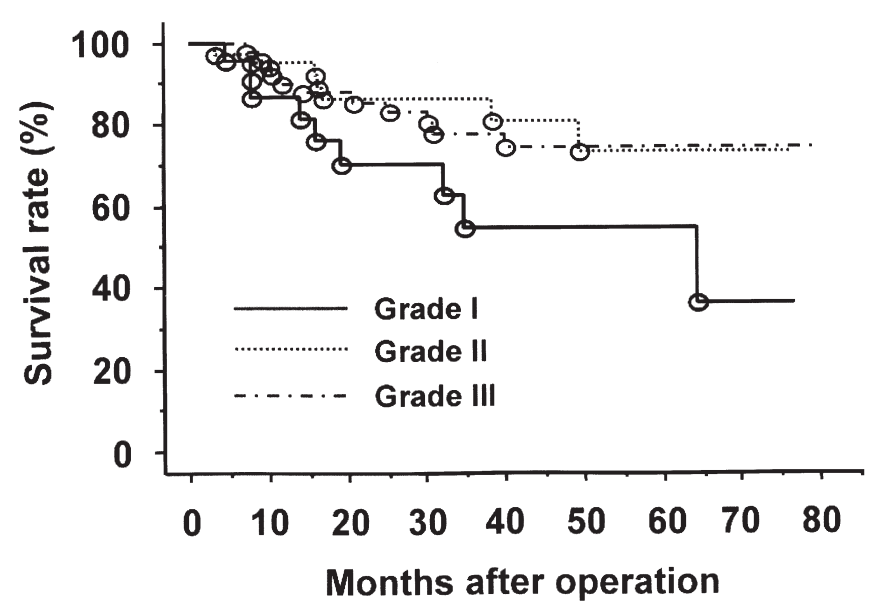

B

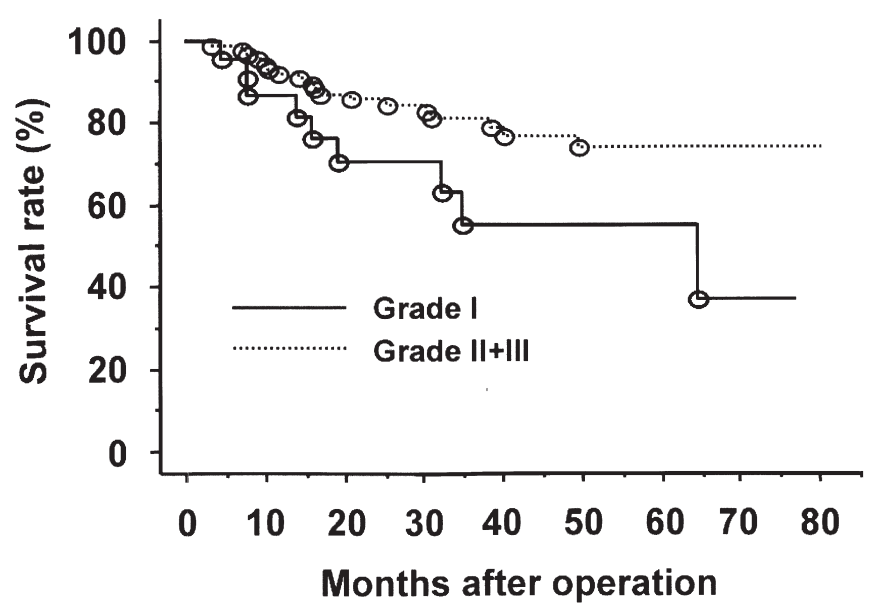

Figure 2. Kaplan-Meier analysis for long-term survival in esophageal squamous cell cancer patients, stratified according to Prdx1 expression. A, comparison of overall survival curves for patients with Prdx1 weak, moderate, and strong tumors $(\mathrm{P}=0.069)$. B, comparison of overall survival curves for patients between Prdx1 weak + moderate and strong tumors $(\mathrm{P}=0.022)$.

between the N0 and N1 groups $(\mathrm{P}=0.048<0.05)$. No significant difference in Prdx1 expression was observed with respect to other factors, such as age, sex, histological grade, and $\mathbf{M}$ factor (Table II).

Survival analysis. The follow-up data of surgically treated ESCC patients was available. For the analyses of the immunohistochemistry results, the tumors with a high $\operatorname{Prdx} 1$ expression were tested against the tumors with a low Prdx 1 expression. A statistical analysis with the log-rank test did not reveal a significant association between the Grade I, Grade II, and Grade III groups (Fig. 2A, $\mathrm{P}=0.069$ ). Although the expression grades were subclassified into 2 groups, Grade I + Grade II and Grade III, a significant association was observed between those groups regarding survival (Fig. 2B, $\mathrm{P}=0.022$ ).

\section{Discussion}

In mammals, the Prdx proteins comprise a highly conserved family of six proteins. All of the Prdx proteins contain a conserved cysteine residue in the $\mathrm{N}$-terminal region that is the active site of catalysis (17). In particular, Prdx 1 is thought to be involved in the redox regulation of the cells and reduce peroxide (18).

Prdx 1 has been thought to act as a tumor suppressor. This idea was derived from the observation that Prdx1 should play a central role in the proliferative signals of two crucial oncoproteins, c-Abl and c-Myc $(4,5)$. Mu et al showed that Prdx 1 overexpression could mimic the growth-promoting activity of c-Myc (5). Egler et al also demonstrated that the $\operatorname{Prdx} 1$ knockout induced ras transformation and the c-Myc target genes had altered expression levels in Prdx1 knockout mice (19). Moreover, the Prdx1 knockout mice generated malignancies in the intestine, lymphomas, and sarcomas with a high frequency (6). In addition, we previously reported that FK228, one of the novel HDAC inhibitors, induced growth inhibition and apoptosis in ESCC (7). HDAC plays a fundamental role in regulating gene expression and chromatin assembly $(9,10)$. Because HDAC inhibitors have different biochemical and biological properties that induce acetylation of the histones, which are the key proteins in the nucleosome and chromatin structure, they are one of the epigenetic drugs that are considered to be most promising as an anti-cancer agent (8-10). Furthermore, HDAC inhibitors may achieve some of the anti-tumor effects through the reaction of a new type of dormant tumor suppressor gene. From these reports and our previous study, we determined by siRNA technology that the activation of $\operatorname{Prdx} 1$ expression is necessary for the effect of FK228 in ESCC. These results imply that Prdx1 is a good candidate for a tumor suppressor.

On the other hand, several reports have suggested that the overexpression of Prdx1 is associated with cancer development (11-15). In fact, Prdx 1 was originally isolated from a rastransformed human mammary epithelial cell, which has 3-fold higher expression of Prdx1 in comparison to an untransformed cell (20). However, so far few reports have attempted to elucidate the molecular mechanism of Prdx1 which correlates with tumor viability. Recently, Zhang et al (21) showed the role of Prdx 1 in protecting cells from ionizing radiationinduced cell death, but this ability was the result of the scavenging function of $\operatorname{Prdx} 1$ and it may not be related to tumor progression.

Prdx 1 gene mutations may contribute to carcinogenesis or tumor growth. However, Gisin et al (22) examined whether the Prdx1 gene is mutated in human hepatocellular carcinoma, and no mutations or polymorphisms of the $\operatorname{Prdx} 1$ gene were found.

In the present study, we detected Prdx 1 expression by immunohistochemistry in tumors from $90 \%$ of 114 ESCC patients. An inverse correlation between the Prdx 1 expression and T-category or stage suggested that Prdx1 may be a key molecule involved with tumor suppression in ESCC. Furthermore, the prognoses of the high-expression Prdx 1 cases were significantly better than those of the low-expression cases. We are now planning our new clinical trial using one of the HDAC inhibitors for esophageal cancer. The Prdx1 expression 
pattern may play an important role in examining the sensitivity of the HDAC inhibitor before treatment.

In conclusion, we have shown here for the first time the correlation between the Prdx1 expression and the clinicopathological parameters in ESCC. We are confident that our results will help in better predicting the prognosis of patients with ESCC.

\section{References}

1. Parkin DM, Bray F, Ferlay J and Pisani P: Global cancer statistics, 2002. CA Cancer J Clin 55: 74-108, 2005.

2. Kurehara $\mathrm{H}$, Ishiguro $\mathrm{H}$, Kimura $\mathrm{M}$, et al: A novel gene, RSRC2, inhibits cell proliferation and affects survival in esophageal cancer patients. Int J Oncol 30: 421-428, 2007.

3. Miller JD, Jain MK, de Gara CJ, Morgan D and Urschel JD: Effect of surgical experience on results of esophagectomy for esophageal carcinoma. J Surg Oncol 65: 20-21, 1997.

4. Wen ST and Van Etten RA: The PAG gene product, a stressinduced protein with antioxidant properties, is an Abl SH3binding protein and a physiological inhibitor of c-Abl tyrosine kinase activity. Genes Dev 11: 2456-2467, 1997.

5. Mu ZM, Yin XY and Prochownik EV: Pag, a putative tumor suppressor, interacts with the Myc Box II domain of c-Myc and selectively alters its biological function and target gene expression. J Biol Chem 277: 43175-43184, 2002.

6. Neumann CA, Krause DS, Carman CV, et al: Essential role for the peroxiredoxin Prdx 1 in erythrocyte antioxidant defence and tumour suppression. Nature 424: 561-565, 2003.

7. Hoshino I, Matsubara H, Hanari N, et al: Histone deacetylase inhibitor FK228 activates tumor suppressor Prdx1 with apoptosis induction in esophageal cancer cells. Clin Cancer Res 11: 7945-7952, 2005

8. Villar-Garea A and Esteller M: Histone deacetylase inhibitors: understanding a new wave of anticancer agents. Int J Cancer 112: 171-178, 2004.

9. Marks P, Rifkind RA, Richon VM, et al: Histone deacetylases and cancer: causes and therapies. Nat Rev Cancer 1: 194-202, 2001.
10. Egger G, Liang G, Aparicio A, et al: Epigenetics in human disease and prospects for epigenetic therapy. Nature 429: 457-463, 2004.

11. Yanagawa T, Ishikawa T, Ishii T, et al: Peroxiredoxin I expression in human thyroid tumors. Cancer Lett 145: 127-132, 1999.

12. Yanagawa T, Iwasa S, Ishii T, et al: Peroxiredoxin I expression in oral cancer: a potential new tumor marker. Cancer Lett 156: $27-35,2000$.

13. Quan C, Cha EJ, Lee HL, Han KH, Lee KM and Kim WJ: Enhanced expression of peroxiredoxin I and VI correlates with development, recurrence and progression of human bladder cancer. J Urol 175: 1512-1516, 2006.

14. Odreman F, Vindigni M, Gonzales ML, et al: Proteomic studies on low- and high-grade human brain astrocytomas. J Proteome Res 4: 698-708, 2005

15. Park JH, Kim YS, Lee HL, et al: Expression of peroxiredoxin and thioredoxin in human lung cancer and paired normal lung. Respirology 11: 269-275, 2006.

16. Johnston PG, Fisher ER, Rockette HE, et al: The role of thymidylate synthase expression in prognosis and outcome of adjuvant chemotherapy in patients with rectal cancer. J Clin Oncol 12: 2640-2647, 1994.

17. Rhee SG, Chae HZ and Kim K: Peroxiredoxins: a historical overview and speculative preview of novel mechanisms and emerging concepts in cell signaling. Free Radic Biol Med 38: 1543-1552, 2005.

18. Wood ZA, Poole LB and Karplus PA: Peroxiredoxin evolution and the regulation of hydrogen peroxide signaling. Science 300 : 650-653, 2003.

19. Egler RA, Fernandes E, Rothermund K, Sereika S, de SouzaPinto N, Jaruga P, Dizdaroglu M and Prochownik EV: Regulation of reactive oxygen species, DNA damage, and c-Myc function by peroxiredoxin 1. Oncogene 24: 8038-8050, 2005.

20. Prosperi MT, Ferbus D, Karczinski I and Goubin G: A human cDNA corresponding to a gene overexpressed during cell proliferation encodes a product sharing homology with amoebic and bacterial proteins. J Biol Chem 268: 11050-11056, 1993.

21. Zhang B, Su Y, Ai G, Wang Y, Wang T and Wang F: Involvement of peroxiredoxin $\mathrm{I}$ in protecting cells from radiationinduced death. J Radiat Res 46: 305-312, 2005.

22. Gisin J, Perren A, Bawohl M and Jochum W: Rare allelic imbalances, but no mutations of the PRDX 1 gene in human hepatocellular carcinomas. J Clin Pathol 58: 1229-1231, 2005. 